当资 Universität

Basel

Philosophisch-Historische

Fakultät

\title{
Frauen und Corona-Proteste
}

Nadine Frei

Ulrike Nack 
(C) 2021

Nadine Frei

Ulrike Nack

Herausgegeben durch / published by:

Seminar für Soziologie, Universität Basel

Seminar for Sociology, University of Basel

Gestaltung / Design: Heidi Franke 


\section{Inhaltsverzeichnis}

Abstract
2

Einleitung 3

Erste Perspektive: Impfungen als Angriff auf die Natur und das Weibliche 5

Zur Neubestimung der Geschlechterverhältnisse 6

Polarisierung der Geschlechtscharaktere und Verwissenschaftlichung der Differenz 7

Romantisches Naturverständnis und Idealisierung des Weiblichen 9

Zweite Perspektive: Die Affirmation der Hoheit über die Sorge um die Angehörigen als Gefährdung der Gesellschaft

Die Sorge um die Angehörigen 12

Der Widerspruch der Trennung von privat und öffentlich 13

Fazit

Referenzen 


\begin{abstract}
Unser Artikel unterbreitet einen theoriegeleiteten Deutungsvorschlag zur Frage, wie die breite Beteiligung von Frauen an den Corona-Protesten verstanden werden kann. Dafür betrachten wir zwei zentrale Themen ihres Protestes: Impfen und Sorge um ihre Angehörigen. Aus einer soziologischen Perspektive zeigen wir auf, inwiefern ihr Protest die für die bürgerlich-kapitalistische Gesellschaft konstitutive vergeschlechtlichte Trennung zwischen privater und öffentlicher Sphäre affirmiert und welche Rolle romantisches Denken darin einnimmt. Die Frauen nehmen die ihnen zugewiesene gesellschaftliche Rolle der sorgenden Frau und des Naturwesens an. Aus einer philosophischen Perspektive beleuchten wir, wie diese Affirmation mit einer Gefährdung der Gesellschaft einhergeht. Diese gründet, wie wir mit Hegel offenlegen, in einem Widerspruch von Frau und Gesellschaft. In ihrem Protest zeigt sich paradigmatisch die dialektische Bewegung von Affirmation und Gefährdung der bürgerlich-kapitalistischen Ordnung.
\end{abstract}

\title{
Schlüsselwörter
}

Corona-Protest, Frauen, Geschlechterverhältnisse, Impfung, Romantik, Hegel

Our article proposes a theoretical interpretation of women's broad participation in the coronavirus protests. For this purpose, we consider two central themes of their protest: vaccination and caring for their loved ones. From a sociological perspective, we show how their protest affirms the gendered division between the private and the public spheres that is constitutive of bourgeois capitalist society, and what role romantic thought plays in this. The women accept the social role assigned to them as caring women and creatures of nature. From a philosophical perspective, we illuminate how this affirmation is accompanied by a threat to society. That threat is based, as we point out with Hegel, on a contradiction of woman and society. Their protest reveals paradigmatically the dialectical movement of affirmation and endangerment of the bourgeois capitalist order.

\section{Keywords}

Corona-virus protest, women, gender relations, vaccination, romanticism, Hegel 


\section{Einleitung}

In der Analyse der Corona-Pandemie und ihren Auswirkungen wird in medialen, aber insbesondere in wissenschaftlichen Debatten von Anfang an auch eine Geschlechterperspektive eingenommen. Bestehende geschlechtliche Ungleichheiten werden durch die coronabedingten Maßnahmen und eine damit im Verbindung stehende wirtschaftliche Krise verschärft (vgl. Kohlrausch und Hoevermann 2020). Frauen sind - aufgrund der horizontalen und vertikalen Segregation in der Berufssphäre - nicht nur von einer stärkeren Arbeitsbelastung u.a. aufgrund veränderter Abläufe, intensiverer emotionaler Beanspruchung und einem erhöhten Infektionsrisiko betroffen, sondern übernehmen auch mehr Verantwortung für die zunehmende Reproduktionsarbeit im privaten Bereich, zum Beispiel im Homeschooling oder der zusätzlichen Kinderbetreuung (vgl. Kohlrausch und Zucco 2020; Villa 2020; Möhring et al. 2020; Petzold et al. 2020). ${ }^{1}$ Trotz der zunehmenden Thematisierung der Auswirkungen auf Frauen im Bezug auf die Corona-Krise fehlt eine geschlechtsspezifische Analyse in der Untersuchung der aktuell in Deutschland, in der Schweiz und in Österreich stattfindenden Corona-Proteste. Wenngleich bislang keine empirische Einzel-Untersuchung zu den Frauen, die an den Corona-Protesten in den deutschsprachigen Ländern teilnehmen, vorhanden ist, so lässt sich zumindest aus den Studien von Nachtwey et al. (2020) und Koos (2021) Folgendes gewinnen: Bei den protestierenden Frauen handelt es sich vornehmlich um bürgerliche Frauen mit höherem Bildungsabschluss und deutscher respektive österreichischer oder schweizerischer Staatsbürgerschaft, die gegenüber esoterischem Denken offen sind, die Impfthematik als relevant ausweisen und ihre Sorge um Kinder ausdrücken.

Wir diskutieren im Folgenden aus zwei eng miteinander verwobenen Perspektiven einer soziologischen und einer philosophischen - heraus die Frage, was diese Frauen dazu bewegen könnte, sich an den CoronaProtesten zu beteiligen. Es handelt sich um eine theoriegeleitete Analyse, deren Thesen empirisch zu prüfen wären. Unser Anliegen ist es, weitere Debatten dieses bislang zu wenig beachteten Themas anzuregen. Für unsere Analyse nehmen wir zwei zentrale Anliegen der Corona-Proteste in den Blick: das Impfen und die Sorge um ihre Angehörigen. Es sind vor allem Frauen, so unsere erste These, die gegen eine, aus ihrer Sicht drohenden, allgemeinen Impfpflicht auf die Straße gehen und die weder sich selbst, noch ihre Kinder, dem Leid oder den möglichen Nebenwirkungen der Impfung aussetzen wollen. Und es sind vor allem Frauen, entsprechend unserer zweiten These, die die Maßnahmen gegen das Coronavirus als leidvolle Erfahrung und Einschränkung ihrer Sorge um ihre Angehörigen wahrnehmen und deswegen gegen sie aufbegehren. Wir deuten ihre Teilnahme an den Corona-Protesten als eine Affirmation der ihnen zugewiesenen gesellschaftlichen Rolle: Sie erfüllen und verteidigen eine romantische Vorstellung von Natur und die ihnen gesellschaftlich zugewiesene Sphäre des Privaten. Diese Affirmation stützt die für die bürgerlich-kapitalistische Gesellschaft konstitutive vergeschlechtlichte Trennung zwischen der privaten und der öffentlichen

Einschränkungen von Frauen mit Migrations- und Fluchthintergrund (über die Auswirkungen der Corona-Maßnahmen für Geflüchtete allgemein vgl. Berger 2020). 
Sphäre. ${ }^{2}$ In der Verteidigung der privaten Sphäre als ihre Sphäre liegt allerdings ein genuin gesellschaftsgefährdendes Moment, welches auch in den Corona-Protesten zum Ausdruck kommt: Indem die Frau eine spezifische Vorstellung von Natur und damit der ihr zugewiesenen privaten Sphäre verteidigt, stellt sie sich gegen die Gesellschaft. Indem sie sich gegen die Maßnahmen zum Schutz der Gesellschaft ${ }^{3}$ ausspricht, gefährdet sie eben jene Gesellschaft, auf deren Rollenzuteilung sie sich in ihrem Protest beruft.

Ziel unserer Überlegungen ist es, eine allgemeine dialektische Bewegung aufzuzeigen, die in den Corona-Protesten der Frauen geradezu paradigmatisch hervortritt: Die für die bürgerlich-kapitalistische Gesellschaft konstitutive vergeschlechtlichte Sphärentrennung birgt trotz Stabilisierung der bestehenden Verhältnisse gleichzeitig eine Gefährdung der Gesellschaft. Wir werden dies aus zwei verschiedenen Perspektiven verdeutlichen. Aus einer soziologischen Perspektive nehmen wir die These, dass manche Frauen Impfen als Angriff auf die Natur und in der Konsequenz auf sich selber sehen, in den Blick. Zur ihrer Untersuchung erfolgt ein Abriss der Neubestimmung der Geschlechterverhältnisse im Übergang zur bürgerlich-kapitalistischen Gesellschaft. Dabei beziehen wir uns insbesondere auf marxistisch- feministische Theorien, welche die kapitalistische Ausdifferenzierung von privater und öffentlicher Sphäre vergeschlechtlicht denken und damit zum Teil auf ältere Debatten innerhalb der Soziologie beziehungsweise den Anfängen der institutionalisierten Geschlechterforschung. Wir betrachten die vergeschlechtlichte Trennung zwischen privater und öffentlicher Sphäre unter den Aspekten einer dichotomen Vorstellung von Natur und Kultur sowie einer Ausdifferenzierung zwischen Heim und Markt. Um zu verstehen, wie es zur gesellschaftlichen Akzeptanz dieser Sphärentrennung gekommen ist, setzen wir uns mit der Romantik auseinander, der nicht nur eine Idealisierung der Natur inhärent ist, sondern auch die Idealisierung von Emotionalität, Mütterlichkeit und Gemeinschaft. Das Vorgehen lässt verstehen, inwiefern Impfen als Angriff auf die Natur aufgefasst werden kann und inwiefern Frauen, die ihre gesellschaftliche Zuweisung in die private Sphäre und die Vorstellung von sich selbst als Naturwesen akzeptieren, Impfen als einen Angriff auf sich selber sehen.

Aus einer philosophischen Perspektive befassen wir uns eingehender mit der Problematik der Sorge um die Angehörigen. Unsere These ist, dass die Affirmation der Rolle der Frau, die durch die vergeschlechtliche Trennung von privater und
2 Wir verstehen unter Kapitalismus einen „übergreifenden gesellschaftlichen Zusammenhang“ (Degele/Winker 2011: 71). Dieser setzt die Akkumulation von Kapital zum höchsten Zweck jeder (Re)produktion. Die kapitalistische Produktionsweise strukturiert die Produktion von Gütern, die soziale Reproduktion und zwischenmenschliche Beziehungsund individuelle Subjektivierungsweisen entlang der Achsen Klasse, Geschlecht und race (Knapp/Klinger 2007). Damit ist nicht gesagt, dass jede

gesellschaftliche Erscheinung durch Kapitalismus determiniert ist, noch dass es keine

gesellschaftlichen Bewegungen geben kann, die sich gegen kapitalistische Strukturierungen richten. Das

Gegenteil ist der Fall. Gleichwohl nehmen wir

Kapitalismus als Grundstruktur moderner

Gesellschaften an, dem vergeschlechtliche und rassistische Ungleichheiten inhärent sind (vgl. Aulenbacher et al. 2012). Vgl. im Weiteren Adamczak 2017; Mies 2015; Scheele/Wöhl 2018).

\footnotetext{
${ }^{3}$ Nicht alle staatlichen Maßnahmen zur Eindämmung der Corona-Pandemie zielen auf die Gesundheit der Mitglieder der Gesellschaft, sondern setzen sich z.B. Wirtschaftsförderung zum Ziel. Auch scheinen manche Maßnahmen, etwa die Abriegelung von Gefängnissen und Geflüchtetenunterkünften, die Gesundheit der direkt Betroffenen eher wenig zu berücksichtigen. In Bezug auf die zentralen Themen der beteiligten Frauen an den Corona-Protesten sind vor allem jene staatlichen Maßnahmen relevant, die unter der Begründung des gesundheitlichen Schutzes der Gesellschaft erlassen werden.
} 
öffentlicher Sphäre geschaffen wird, eben jene Gesellschaft gefährden kann, die auf dieser Trennung beruht. Wir deuten die Beteiligung von den Frauen an den CoronaProtesten, die aufgrund der Sorge um ihre Angehörigen protestieren, als Verteidigung ihrer Hoheit über die private Sphäre. ${ }^{4}$ In der Verteidigung der Sorge um ihre Angehörigen stellen sie sich zum vermeintlichen Schutz ihrer Nächsten gegen die staatlichen Maßnahmen zum Schutz der Gesellschaft. Unter Rückgriff auf Hegels Überlegungen zum Verhältnis von Frau und Gesellschaft entwickeln wir die Deutung, dass diese Gefährdung auf dem Widerspruch der Trennung von privater und öffentlicher Sphäre beruht und dahingehend den bürgerlich-kapitalistischen gesellschaftlichen Verhältnissen geradezu eingeschrieben ist.

\section{Erste Perspektive: Impfungen als Angriff auf die Natur und das Weibliche}

Im Zusammenhang mit der Corona-Pandemie kristallisiert sich eine Haltung heraus, die als Kritik gegen eine Impfpflicht zur Bekämpfung des Corona-Virus formuliert wird (vgl. Frei et al. 2021: 253), aber im Grunde eine ablehnende Haltung gegenüber Impfungen generell meint. ${ }^{5}$ Impfkritik ist zwar kein neues Phänomen, ${ }^{6}$ jedoch ergeben sich für Impfgegner:innen mit den aktuellen Corona-Protesten Anknüpfungsmöglichkeiten, diese Kritik im Rahmen

\footnotetext{
${ }^{4}$ Exemplarisch zur Zurückweisung von staatlichen Maßnahmen und die Verteidigung des elterlichen Hoheitsgebietes siehe die Website

www.elternstehenauf.de (Stand 21.04.2021).

${ }^{5}$ Ihre Kritik ist nicht begründet in einer staatlich angekündigten Impfpflicht, vielmehr wird eine solche von ihnen behauptet. Es geht in unseren

Überlegungen also nicht um Impfskeptiker:innen im Allgemeinen, sondern um Impfgegner:innen, die ihre Ablehnung von Impfungen in einem rechtsoffenen Protest artikulieren.

${ }^{6}$ Das Impfen hat eine lange kontroverse Geschichte, deren Anfang sich ins 18. Jahrhundert datieren lässt
}

eines größeren Protestes auf die Straße zu tragen. Eine Idealisierung der Stärke des Immunsystems, naturgegebene (Selbst)Heilungskräfte, die Natürlichkeit der eigenen Immunkraft sowie die Reinheit des Körpers werden der Unnatürlichkeit und Künstlichkeit, die dem Impfen und den Pharmaunternehmen attestiert werden, gegenüber gestellt. In diesem Kontext diskutieren wir aus einer historisch-soziologischen Perspektive folgende These: Manche Frauen nehmen das Impfen als Angriff auf die Natur im Allgemeinen und auf das Weibliche im Besonderen wahr.

Die gesellschaftliche Zuweisung der Frau als Naturwesen auf die private Sphäre und die damit verknüpfte Vorstellung des Weiblichen sind moderne Entwicklungen, die zwar seit ihrem Ursprung Veränderungen unterworfen sind, denen jedoch eine gewisse Kontinuität eigen ist, wie wir am Beispiel der Corona-Proteste argumentieren. Um dem geschlechtsspezifischen Zusammenhang zwischen der privaten und der öffentlichen Sphäre, Natur und Kultur sowie Heim und Markt nachzugehen, blicken wir zunächst auf die Neubestimmung der Geschlechterverhältnisse im Übergang zur bürgerlich-kapitalistischen Gesellschaft. Wir konzentrieren uns dabei auf drei Momente: Erstens skizzieren wir die mit der Entstehung des Kapitalismus einhergehende Ausdifferenzierung zwischen

(vgl. Krüger/Krüger 2015: 100). Reich rekonstruiert in ihrer qualitativen soziologischen Studie über das Impfen in den USA Begründungen von Eltern, mit denen diese ihre ablehnende Haltung gegenüber Impfungen rechtfertigen. Nach Reich verstehen sich Eltern als Expert:innen ihrer eigenen Kinder, weshalb sie ihrem eigenen Urteil mehr vertrauen als "qualifizierten " Expert:innen (vgl. Reich 2016.: 67ff.). Sie lehnen Impfungen mit der Begründung ab, dass diese ein künstlicher Eingriff gegenüber der natürlichen Immunkraft darstellen (vgl. Reich 2016: 97ff.). Zudem hegen sie ein tiefliegendes Misstrauen gegenüber Pharma-Unternehmen (vgl. Reich 2016: 118). 
Heim und Markt. ${ }^{7}$ Zweitens fokussieren wir uns auf die Etablierung der Vorstellung einer grundlegenden Andersartigkeit von Frauen und Männern, mit der eine Abkehr von einer aristotelischen und christlichen Tradition einhergeht, in der eine Inferiorität der Frau in ihrem Defizitcharakter zum Mann gesehen wurde (vgl. Badinter 1984: 20f.). Die neue, nun natürliche ${ }^{8}$, Legitimation der Geschlechterverhältnisse versucht, im modernen Geschlechterverhältnis den „vernünftige[n] Plan und Zweck der Natur" (Hausen 2001: 169) wissenschaftlich nachzuzeichnen. Verschiedene Wissenschaften - wir gehen veranschaulichend auf die damals noch junge Soziologie ein interessieren sich für die Geschlechterdifferenz, um diese gleichsam zu legitimieren. Drittens wird die Bedeutung der Romantik mit ihrem spezifischen Naturverständnis in den Blick genommen. Die Romantik spielte die Rolle der bejahenden Ideologie, welche die Neubestimmung der Geschlechterverhältnisse nicht nur begrüßte, sondern in einer Weise verklärte, die die (gewaltvollen) gesellschaftlichen Verhältnisse außer Acht lässt. Dies lässt sich anhand zweier Aspekte im romantischen Denken gut zeigen: erstens der Trennung von Natur und Kultur, welche die Reinheit der Natur behauptet und diese idealisiert, um im gleichen Atemzug die Künstlichkeit der Kultur abzulehnen. Zweitens anhand der normativen Unterscheidung zwischen Gemeinschaft und Gesellschaft, wobei die private Gemeinschaft der anonymen Gesellschaft vorzuziehen sei. Die der Natur und der Gemeinschaft zugesprochenen Werte wurden in der Romantik idealisiert und erhöht - und gleichzeitig damit die Frau, der als "Naturwesen« der Bereich »Gemeinschaft«, also der privaten Sphäre,

\footnotetext{
${ }^{7}$ Wobei anzumerken ist, dass die Trennung zwischen privater und öffentlicher Sphäre nicht einfach auf Heim und Markt zu verkürzen ist, auch wenn wir diese Begriffspaare im Folgenden eng verknüpft verwenden.
}

zugewiesen wurde. Die natürliche und umsorgende Weiblichkeit wurde im romantischen Denken ein Ideal. Die Ausdifferenzierung von Heim und Markt, die mit einer binären und hierarchischen Vorstellung der Geschlechter einherging und die im romantischen Denken idealisiert wurde, bietet (noch) heute eine Grundlage zum Verständnis der Impfablehnung, welche Frauen an den Corona-Protesten zum Ausdruck bringen. Erst die Akzeptanz der Vorstellung des romantisierten dualistischen Geschlechterverhältnisses, welches Frauen zu Naturwesen stilisiert, macht plausibel, wie Impfungen von manchen Frauen als Angriff auf die Natur und auf den eigenen, natürlichen Körper verstanden werden können.

\section{Zur Neubestimung der Geschlechterverhältnisse}

Im Folgenden skizzieren wir, um die Neubestimmung der Geschlechterverhältnisse im Übergang zur bürgerlich-kapitalistischen Gesellschaft im Kontext einer vergeschlechtlichten Sphärentrennung zu verorten, die historische Ausdifferenzierung zwischen Markt und Heim mit Marx und daran anknüpfend Federici. Marx analysiert den jahrhundertelangen Übergang von der feudalen in die bürgerlich-kapitalistische Gesellschaft mit Fokus auf die Produktions- und Eigentumsverhältnisse und die damit verbundenen Konsequenzen für die Gesellschaftsordnung, welche die Sphärentrennung von Heim und Mark einschließt. Er wendet sich gegen liberale Ökonomen wie Smith und Ricardo, die mit ihren „Robinsonaden“ (MEW 23: 90) Positiverzählungen des Kapitalismus entworfen hätten, nach denen dieser zum Wohle aller

8 "Natürlich" meint hier einen Begründungsmodus, der durch den Rückgriff auf die Vorstellung einer unveränderbaren und gegebenen Natur die Geschlechterdifferenz als selbstverständlich und unabänderlich auszuweisen sucht. 
entstanden sei. Im Gegensatz zum liberalen Fortschrittsenthusiasmus, welcher das Freiheitsmoment gegenüber der feudalen Leibeigenschaft hervorhebt, verweist Marx auf die Gewaltförmigkeit der bürgerlichkapitalistischen Gesellschaft. Im Zuge der „sogenannte[n] ursprüngliche[n] Akkumulation“ (MEW 23: 741) werden die von ihren Produktionsmitteln getrennten Produzent:innen gezwungen, ihre Arbeitskraft auf dem Arbeitsmarkt zu verkaufen. Marx spricht vom „historischen Scheidungsprozess von Produzent und Produktionsmittel“ (MEW 23: 742) als grundlegende Voraussetzung für die Entstehung des Kapitalismus, mit dem eine Trennung von Produktions- und Reproduktionssphäre, also von Heim und Markt, einhergeht. Federici orientiert sich in ihrer Akzentuierung der Neubestimmung der Geschlechterverhältnisse an Marx, kritisiert jedoch seine Geschlechtsblindheit: Nicht nur die Enteignung der existentiellen Grundlagen der Bäuer:innen sei für die Herausbildung des Kapitalismus notwendig gewesen, sondern ebenso die „Zerschlagung der Macht der Frauen“ und das Vorantreiben ihrer „Unterordnung unter die Erfordernisse der Arbeitskraftproduktion“ (Federici 2015: 82). ${ }^{9}$

\footnotetext{
${ }^{9}$ Sie sieht darin die Erklärung für die Ermordung hunderttausender Frauen während der

Hexenverfolgungen (vgl. Federici 2015: 206). Darin, "der Kontrolle, die Frauen über ihren Körper und die Reproduktion ausgeübt hatten, ein Ende zu setzen", sieht sie einen "genuinen Krieg gegen die Frauen" (Federici 2015: 111).

${ }^{10}$ Wir werden zwar nachzeichnen, auf welche Weise durch vergeschlechtlichte Sphärentrennung zwischen Heim und Markt die moderne patriarchale Ordnung legitimiert wird, allerdings sei an dieser Stelle erwähnt, dass im Übergang zur kapitalistischen Gesellschaft Momente zu finden sind, in denen der Einflussbereich von Frauen bedeutsam war. In der frühindustriellen Arbeitsorganisation löste die sich verbreitende Heimindustrie allmählich die Landwirtschaft ab, wodurch die häusliche Sphäre und die Arbeit von Frauen zunächst an Bedeutung gewannen. In der Familienökonomie hatten Frauen dabei eine zentrale Funktion, insofern ihre Macht auf Produktion und direkter Kontrolle von

lebensnotwendigen Entscheidungen beruhte. Diese Bedeutung nahm aber im Zuge der Industrialisierung und des Niedergangs der Heimarbeit ab. Zu
}

Nicht nur die Entmachtung der Frauen und ihre Anpassung an die Arbeitskraftproduktion geschehe gewaltvoll, auch ihre Zuweisung zu der Sphäre des Weiblichen - der Sphäre des Privatem bzw. des Heimes - sei ein Akt der Gewalt. Mitunter aufgrund dieser gewaltvollen Bewegung stellt sich die Frage, auf welche Weise die Zuweisung der Frau zur privaten Sphäre/dem Heim und die Zuweisung des Mannes zur öffentlichen Sphäre/dem Markt gesellschaftlich legitimiert wird $?^{10}$ Um dieser Frage nachzugehen, charakterisieren wir im Folgenden die wissenschaftliche "Entdeckung« der Natur und die "Erfindung» des Weiblichen.

\section{Polarisierung der \\ Geschlechtscharaktere und \\ Verwissenschaftlichung der Differenz}

Die vergeschlechtlichte Ausdifferenzierung zwischen Heim und Markt wird begleitet von einer wissenschaftlich begründeten Vorstellung von der grundlegenden Andersartigkeit von Frauen und Männern. Hausen (2001) macht den ideologischen Ursprung polarisierter Geschlechtscharaktere im 18. Jahrhundert aus. Die Idee der Geschlechtscharaktere $^{11}$, womit eine je

beobachten war vielmehr eine Trennung in den finanziell bedeutsamen außerhäuslichen Bereich, in welchem zunehmend nur Männer tätig waren und die sozial weniger bedeutsame Hausarbeit, welche vor allem von Frauen erledigt wurde (vgl. Honegger und Heintz 1984: 14ff.; auch Mies 2015).

${ }^{11}$ Hausen ausführlicher: „Die variationsreichen Aussagen über "Geschlechtscharaktere" erweisen sich als ein Gemisch aus Biologie, Bestimmung und Wesen und zielen darauf ab, die "naturgegebenen ", wenngleich in ihrer Art durch Bildung zu vervollkommnenden Gattungsmerkmale von Mann und Frau festzulegen. Den als Kontrastprogramm konzipierten psychischen

"Geschlechtseigenthümlichkeiten « zu Folge ist der Mann für den öffentlichen, die Frau für den häuslichen Bereich von der Natur prädestiniert. Bestimmung und zugleich Fähigkeiten des Mannes verweisen auf die gesellschaftliche Produktion, die der Frau auf die private Reproduktion. Als immer wiederkehrende zentrale Merkmale werden beim Manne die Aktivität und Rationalität, bei der Frau die Passivität und Emotionalität hervorgehoben“

(Hausen 2001: 166). 
spezifische Natur gemeint ist bzw. wodurch das „Wesen von Mann und Frau“ (Hausen 2001: 162) erfasst werden soll, ist eine moderne Erfindung. Die Vorstellung, dass Männer und Frauen „Träger und Trägerinnen qualitativ anderer Charaktere“ (Connell 2000: 88) sind, löst eine bis dahin vorherrschende Vorstellung von einem Defizitcharakter von Frauen ab: „Frauen werden zwar als unterschieden von Männern wahrgenommen, aber im Sinne unvollkommener oder mangelhafterer Exemplare des gleichen Charakters (zum Beispiel mit weniger Vernunft begabt)“ (Connell 2000: 88). Natürliche Begründungen lösen ständische und religiöse ab (vgl. Hausen 2001: 167f.; Badinter 1984: 18). Mit dieser modernen Legitimierung der Geschlechterverhältnisse findet eine „Verwissenschaftlichung der Differenz“ (Honegger 1989: 146) statt. Es sind zwar unterschiedliche Wissenschaften, die sich der neu entdeckten Geschlechterdifferenz widmen, gemein ist ihnen jedoch, die „Ordnung der Geschlechter" als "getreue[s] Abbild der natürlichen Ordnung der Dinge“ (Honegger 1989: IX) darzulegen. Im Bezug auf die „Entdeckung des Weibes“ (Honegger 1989: 147), die sie in das späte 18. Jahrhundert datiert, stellt Honegger fest, dass „die Naturwissenschaften das scheinbar objektive Arsenal abgeben, aus dem sich Alltagswissen wie systematisierendes Denken großzügig bedienen“ (Honegger 1989: 149). Soziologische Erklärungen tragen das Ihrige dazu bei. Dies zeigt sich etwa an den Überlegungen zweier zentraler Begründer der Soziologie. Durkheim sieht die Frau als weniger differenziert an, sie sei „ein viel instinktiveres Wesen [...] als der Mann“, ihr ,geistiges Leben ist weniger entwickelt" (Durkheim 2014: 313). Mit einer ähnlichen Begründung setzt er sich gegen aufkommende Emanzipationsbestrebungen zur Wehr: „Wer für die kommenden Zeiten für die Frau dieselben Rechte verlangen will wie für den Mann, übersieht viel zu sehr, dass das Werk von Jahrhunderten nicht in einem Augenblicke abgeschafft werden kann, und dass im übrigen diese formelle Gleichheit solange nicht rechtens sein kann, als die psychologische Ungleichheit so eklatant ist" (Durkheim 2014: 458). Simmel dagegen ist um eine Gleichbewertung der Geschlechterrollen bemüht, die die Geschlechterdifferenz aber gleichzeitig bestätigt. Mit der Sphärentrennung von Markt und Hauswirtschaft sei eine „schärfere Arbeitsteilung zwischen den Geschlechtern“ (GSG 6: 512) zu beobachten: „Aus sehr naheliegenden Ursachen fällt der Frau die nach innen, dem Manne die nach außen gewandte Tätigkeit zu“ (GSG 6: 512). Das Tätigkeitsfeld der Frau sei von der „Natur her“, also durch „das Tragen und die Pflege der nächsten Generation“ (Simmel 1985: 177), bestimmt. Der Mann hingegen müsse sich sein Tätigkeitsfeld erst suchen, der „Mangel eines naturgegebenen Tätigkeitsinhalts wies ihn auf schöpferische Freiheit“ (Simmel 1985: 177) hin. Simmel sieht die Frau zwar ebenso wie Durkheim als weniger differenziert als den Mann an - sie hänge „wirklich noch enger und tiefer [...] mit dem dunkeln Urgrund der Natur zusammen“ (GSG 6: 516) -, jedoch betont er die Gleichrangigkeit und Komplementarität der Geschlechterrollen. Mit dem Verweis auf die Komplementarität, im Sinne eines harmonischen Gefüges, trägt er zur gesellschaftlichen Akzeptanz dieser vergeschlechtlichten Zuweisung und damit zur modernen patriarchalen Ordnung bei.

Obwohl diese moderne Legitimierung der Geschlechterverhältnisse mitunter gegen Emanzipationsbestrebungen von Frauen eingesetzt wurde (vgl. Hausen 2001: 172), blieb selbst die erste Frauenbewegung von der Kernidee nicht unberührt. Zum 
Beispiel beruft sich Marianne Weber, eine wichtige Figur der bürgerlichen Frauenbewegung, auf Simmels komplementäres Verständnis der Geschlechterrollen (vgl. Weber 2008). Aber nicht nur die bürgerliche Frauenbewegung transportiert diese Vorstellung. In der proletarischen Frauenbewegung zeigt sich, trotz anderweitiger Differenzen, eine Akzeptanz der Vorstellung vom Weiblichen. So findet sich etwa bei Zetkin der Gedanke, dass „Frauenagitation“ (Zetkin 2008: 199) für den Sieg des Sozialismus zwar unabdingbar und daher stark zu fördern sei, diese jedoch nicht auf Kosten der „Pflichten als Mutter und Gattin“ (Zetkin 2008: 198) geschehen dürfe. ${ }^{12}$ Es lässt sich festhalten, dass die Neubestimmung der Geschlechterverhältnisse mit einer spezifischen Vorstellung des Weiblichen, also ihren Charaktereigenschaften und den damit verknüpften idealtypischen Sphären, verwoben sind. Wir zeigen im Folgenden, warum die Romantik durch ihre Idealisierung und Überhöhung der Natur und des Weiblichen eine wichtige Rolle für die Akzeptanz der neu begründeten Geschlechterverhältnisse spielt und wie manche ihrer Ideale sich noch heute in den Corona-Protesten ${ }^{13}$ der Frauen niederschlagen.

\section{Romantisches Naturverständnis und Idealisierung des Weiblichen}

Die Romantik, verstanden als geistesgeschichtliche Bewegung mit großer Bedeutung von 1750 bis 1850, ist auf das Engste verbunden mit den zeitgleich hervortretenden Phänomenen von Industrialisierung, Rationalisierung, Individualisierung, Aufklärung und Säkularisierung, indem sie insbesondere auf negativ empfundene Tendenzen und Gefahren dieser Momente der Moderne verweist. Sie bringt in ihrer Kritik an der Aufklärung veränderte Werte und Normen sowie Formen des Denkens und Erlebens hervor (vgl. u.a. Berlin 1999; Mannheim 1984). Gegenstand der romantischen Kritik ist eine zunehmende Berechenbarkeit, Sachlichkeit, Rationalisierung. Dem ,analytische[n], zergliedernde[n] und zerteilende[n] Denken“ (Klinger 1992: 226) der als "kalt" wahrgenommenen Gesellschaft wird eine Einheitlichkeit und Ganzheit, die in der "warmen" Gemeinschaft zu suchen und zu finden ist, gegenübergestellt. Wichtige Bezugspunkte im romantischen Denken sind ein „nichtinstrumentelle[s] Naturverhältnis“ (Klinger 1992: 230), die Überhöhung der Natur und die Vorstellung ihrer Reinheit als utopischer Fluchtpunkt in der als dekadent und zerstörerisch empfundenen Moderne. Zum romantischen, die Natur verklärenden Narrativ gehört seit Beginn der Moderne die Auflehnung gegen die (technische) Zivilisation und eine Sehnsucht nach Einheit in der Gemeinschaft. Kulturpessimistisch wird eine wertende Differenzierung zwischen Natur und Kultur vorgenommen. Diese bringt eine spezifische Vorstellung von Weiblichkeit und eine Stilisierung der Mütterlichkeit mit sich (vgl. Hausen 2001: 172). ${ }^{14}$ Für die Neubestimmung der Geschlechterverhältnisse wird eine Idealisierung von Weiblichkeit in Form von

\footnotetext{
12 Allerdings gibt Badinter zu bedenken, dass es in der Akzeptanz der geschlechtlichen Arbeitsteilung auch um Folgendes ging: "Sie glaubten an die Versprechungen und dachten, Anrecht auf die Achtung der Männer, Anerkennung ihrer Nützlichkeit und ihrer Eigenart zu erlangen" (Badinter 1984: 114).

${ }^{13} \mathrm{Vgl}$. Frei et al. $2021 \mathrm{zu}$ den romantischen Motiven innerhalb der Corona-Proteste.
} 
Mütterlichkeit und Emotionalität, die Vorstellung einer feindlichen Außenwelt (Gesellschaft) und eines friedlichen Heims (Gemeinschaft) konstitutiv (vgl. Honegger und Heintz 1984: 26). Die Rolle des romantischen Denkens in der sich für die bürgerlich-kapitalistische Gesellschaft typisch erweisenden Trennung zwischen privater und öffentlicher Sphäre liegt damit in der Überhöhung der Rolle der Frau. In der romantisch inspirierten Suche nach Einheit und Ganzheitlichkeit kommt der Idealisierung des Weiblichen und dem, was ihr zugeschrieben wird, eine wichtige Rolle zu: Idealisiert werden ein spezifisches Konzept von Weiblichkeit, das Private als weibliche Sphäre sowie der weibliche Körper mit seiner »lebensspendenden« Kraft.

Dieser historische Abriss über die Neubestimmung der Geschlechterverhältnisse erlaubt zu verstehen, wie das Deutungsmuster der Geschlechterdifferenz eine vergeschlechtlichte Trennung zwischen privater und öffentlicher Sphäre unterstützt. Er skizziert zudem, auf welche Weise die Vorstellung einer weiblichen Sphäre mit der Vorstellung einer normativen Trennung von Natur und Kultur verbunden wird. Zwar haben sich die Geschlechterverhältnisse seit dem Übergang in die bürgerlichkapitalistische Gesellschaft grundlegend verändert und Frauen sind nicht zwingend ausschließlich der privaten Sphäre verhaftet, doch gibt es nach wie vor Frauen, die ihre gesellschaftliche Zuweisung zur privaten Sphäre und der Natur, wie wir sie historisch nachgezeichnet haben, affirmieren. ${ }^{15}$ Betrachtet man nun die Argumente gegen das Impfen (vgl. Reich 2017), zeigt sich die romantisch inspirierte Vorstellung einer Natürlichkeit der Selbstheilungskräfte, die mit einer Künstlichkeit von Medizin kontrastiert wird. In dieser Vorstellung können Impfungen, so wie sie bei den Corona-Protesten als drohende Pflicht behauptet werden, als Angriff auf die Natur verstanden werden. Das Impfen wird zum artifiziellen Akt, der sich gegen eine mit sich selbst im Einklang gedachte Natur richtet. Verteidigen nun Frauen mit ihrem Protest eine romantisch verstandene Natur im Allgemeinen und das ebenso verstandene Weibliche im Besonderen, dann affirmieren sie paradigmatisch die bürgerlichkapitalistische Sphärenzuweisung. Diese Affirmation motiviert Frauen dazu, ihren und andere Körper gegen Eingriffe von außen zu verteidigen. ${ }^{16}$ Im Falle des Impfens bedeutet die Verteidigung dieser Körper jedoch die Gefährdung anderer. ${ }^{17}$ Darin besteht ein ambivalentes Spannungsverhältnis zwischen der Frau, die sich auf Natur und Weiblichkeit beruft, und der Gesellschaft, deren Mitglieder gesundheitlich gefährdet sind. Eine Entscheidung gegen eine Impfung kann eine Entscheidung gegen das Wohl der Gesellschaft darstellen. Dieses dialektische Moment der vergeschlechtlichten Sphärentrennung vertiefen wir im Folgenden am Beispiel der Sorge um die Angehörigen.
${ }^{15}$ Vgl. zu den Persistenzen wie auch zum Wandel u.a. Becker-Schmidt und Knapp 1987; Winker 2007; Lenz 2013; Walgenbach 2015; Fraser 2016; von Alemann et al. 2017; Alischer 2018; Rendtorff et al. 2019.

Beispielsweise argumentiert Soiland, dass seit dem Beginn des neoliberalen Umbaus in den 1970er Jahren Frauen zwar vermehrt einer Lohnarbeit nachgingen, ihnen jedoch die unbezahlte

Reproduktionsarbeit weiterhin zukam, wodurch die Enteignung ihrer Lebenskraft und -zeit

fortgeschrieben wird. Sie analysiert den neoliberalen Umbau als "Neue Landnahme" und weist auf den „stillschweigend vor sich gehenden
Ressourcentransfer [...] aus den Haushalten und damit aus dem Care-Sektor" in Kapital hin (Soiland 2018: 107; vgl. auch 2019).

\footnotetext{
${ }^{16}$ Hier kommt durchaus ein

Selbstbestimmungsmoment der Frau zum Ausdruck.

17 Impfungen, wie die gegen das Coronavirus, dienen nicht nur dem "individuellen Krankheitsschutz", sondern auch dem „Populationsschutz" (Meyer et al. 2002: 323).
} 
Zweite Perspektive: Die Affirmation der Hoheit über die Sorge um die Angehörigen als Gefährdung der Gesellschaft

Die Impfkritik der Frauen, die an den Corona-Protesten teilnehmen, beinhaltet also nicht nur eine Affirmation ihrer gesellschaftlichen Rolle, sondern kann sich auch gegen die Gesellschaft richten. In diesem Abschnitt wollen wir diese doppelte Bewegung von Affirmation der bürgerlich-kapitalistischen vergeschlechtlichen Sphärentrennung und Gefährdung der Gesellschaft noch einmal genauer untersuchen und dabei den Gefährdungsaspekt betonen. Diesen sehen wir in einem Widerspruch von Frau und Gesellschaft begründet, der sich aus der Sphärentrennung ergibt.

Mehr noch als in der Impfthematik tritt dieser Aspekt vielleicht zutage, wenn wir einen weiteren Beweggrund betrachten, der Frauen an den Corona-Protesten teilnehmen lässt, und den wir begrifflich als "Sorge um die Angehörigen" fassen. Wir wollen in diesem Abschnitt zeigen, dass Frauen, denen die gesellschaftliche Rolle des Erhalts der Gesellschaft durch Erziehung ihrer Kinder und Sorge um ihre Angehörigen zukommt, sich durch eben jene Tätigkeiten gegen die Gesellschaft wenden können. Abstrakter ausgedrückt: Die gesellschaftliche Trennung in private und öffentliche Sphäre birgt immer die Gefahr des Agierens des Privaten gegen das Öffentliche.

In der Einleitung haben wir kurz aufgezeigt, wie sich die Maßnahmen gegen das Coronavirus auf Frauen auswirken. Sie werden zweifach getroffen: Zum einen bedeuten vor allem Maßnahmen, welche die Lohnarbeit umstrukturieren, eine zusätzliche psychische, gesundheitliche und ökonomische Belastung von Frauen. Zum anderen greift der Staat durch Maßnahmen wie Schul- und Kindergartenschließungen, Masken- und Abstandspflicht und Besuchsverbote in Heimen in Bereiche der Pflege und Erziehung der Angehörigen ein, also in Bereiche, die in der derzeitigen Gesellschaft primär Frauen unterliegen. Coronabedingte Maßnahmen führen also einerseits zu mehr Druck auf Frauen und gefährden andererseits einen ihrer Hoheitsbereiche, nämlich den der Sorge um die Angehörigen. Aus dieser zweifachen Betroffenheit lassen sich zwei Beweggründe erschließen, die Frauen zum Protest gegen die Corona-Maßnahmen anhält: ein Aufbegehren gegen die Mehrbelastung und ein Aufbegehren gegen den Entzug ihres Hoheitsbereiches. Von diesen zwei Beweggründen interessiert uns hier allein der zweite, da wir vermuten, dass die Frauen, die unter der zusätzlichen und lohnarbeitsbedingten Belastung leiden, eher weniger, jene Frauen hingegen, die sich vornehmlich um ihre Angehörigen sorgen, eher mehr dazu neigen, an diesen Protesten teilzunehmen. ${ }^{18}$ Durch Rückgriff auf schon Gezeigtes und unter Zuhilfenahme Hegels zeigen wir auf, inwiefern die bürgerlich-kapitalistische vergeschlechtliche Trennung in eine private und eine öffentliche Sphäre als mitursächlich für die breite Beteiligung von Frauen an den Corona-Protesten zu denken wäre und wie die Proteste selbst auf die Flexibilität dieser Trennung und

selbstverantwortliche Individuum gegen staatliche Zwänge in Szene setzen. Gleichzeitig ist es mitunter eben dieser Charakterzug, der die Frauen, die ihre Identität auf das Private aufbauen, zu den Protesten zieht. 
ihrem inhärenten Widerspruch aufmerksam machen.

\section{Die Sorge um die Angehörigen}

Was meinen wir nun, wenn wir schreiben, die Corona-Maßnahmen gefährden den Hoheitsbereich der "Sorge um die Angehörigen«? Frauen tragen nach wie vor die Hauptlast in der Reproduktionsarbeit. Sei es bezahlt als Beruf oder unbezahlt im Privaten, sie sind es, die vornehmlich Kinder erziehen, Menschen pflegen und emotionale Arbeit verrichten (vgl. u.a. Neumann/Winker 2019; Dreas 2019; Riegraf 2019; Hobler et al. 2020). In Bezug auf Corona sind auch sie es, die plötzlich von Besuchen bei Pflegebedürftigen ausgeschlossen sind, aber gleichzeitig an deren Einsamkeit partizipieren, oder deren Kinder nur noch verunsichert, wenn überhaupt, zur Schule gehen. Man könnte nun die Anteilnahme am Leid ihrer Angehörigen als Protestbeweggrund verstehen, wäre da nicht die Besonderheit, dass die Sorge um ihre Angehörigen in den CoronaProtesten einen Ausdruck in Forderungen findet, die sich gegen Maßnahmen richten, die doch gerade ihre Angehörigen schützen sollen. Maßnahmen wie Masken- und Abstandsregeln und Besuchsverbote bzw. reglementierungen sollen gesundheitliches Leid vermeiden und werden dennoch als Leid verursachend abgelehnt. Es ist dieser Punkt, der uns vermuten lässt, dass die Sorge um ihre Angehörigen nicht primär die Angehörigen betont, sondern die Sorge selbst. Unserer Ansicht nach geht es in den Corona-Protesten weniger um das Leid der Angehörigen, als vielmehr um die Frage, wer sich wie um dieses Leid zu sorgen hat: der Staat oder die Frau?

19 Von den Anfängen einer Etablierung der strikten Geschlechterteilung über einen Sozialstaat, der Frauen auch in der Öffentlichkeit "weiblichen Tätigkeiten « nachgehen ließ, hin zu neoliberalen
Indem Frauen das Leid ihrer Angehörigen, welches die Corona-Maßnahmen verschulden, anprangern, bringen sie eine Erfahrung ihres Aufgaben- und damit ihres Wertverlustes zum Ausdruck: Frauen dürfen sich aufgrund der Corona-Maßnahmen nicht mehr so um ihre Angehörigen kümmern, wie sie es gewohnt sind. Sie dürfen ihre Angehörigen in Heimen, wenn überhaupt, nur eingeschränkt besuchen. Bei Schul- und Kindergartenschließungen haben sie ihre Kinder zu Hause zu unterrichten und gleichzeitig sorgende Mutter und strenge Lehrerin zu sein. Der Staat schreibt ihnen vor, die Kontakte ihrer Kinder stark einzuschränken und sie ggf. Maske tragen $\mathrm{zu}$ lassen. In diesem Sinne entziehen Corona-Maßnahmen den Frauen die Hoheit über den Bereich der Pflege und der Sorge. Auf jene Bereiche, die Frauen in allen patriarchalen Gesellschaften zugeschrieben werden, jedoch gründet sich ihre gesellschaftliche Rolle. Überspitzt gesagt haben die Frauen, die ihr Selbstwertgefühl primär auf diese Rolle der Sich-um-anderesorgenden Frau aufgebaut haben und die sich durch staatliche Maßnahmen in ihrer Rollenausübung gehindert sehen, aus ihrer Sicht allen Grund, gegen die Corona-Maßnahmen zu protestieren: Sie bedrohen ihre gesellschaftliche Existenz. Ihre gesellschaftliche Rolle steht immer dann auf dem Spiel, wenn die Ausführung ihrer Sorge um ihre Angehörigen gefährdet ist.

In diesem Beweggrund zeigen sich zwei zusammenhängende Momente. Zum einen kommt in ihm ein Ringen um die Ausgestaltung der Trennung von privater und öffentlicher Sphäre zum Ausdruck, was nicht nur daran erinnert, dass die Trennung veränderbar ist, sondern auch daran, dass sie immer wieder verändert worden ist. ${ }^{19}$ Sie

Etablierungen, die Frauen die Organisation des Privaten ließ und diese zusätzlich als (oft prekäre) Arbeitskraft etablierte. 
ist in dieser Hinsicht genuin flexibel. Zum anderen offenbart er einen Widerspruch, der dieser Trennung eingeschrieben ist: Im Zweifel sind Familie und Gesellschaft, Privates und Öffentliches, nicht nur unvereinbar, sondern gegeneinander gerichtet. Dieser Widerspruch, auf den wir im nächsten Abschnitt genauer eingehen, tritt besonders deutlich in Zeiten der Verschiebung der Trennungslinie, wie sie mit den derzeitigen Corona-Maßnahmen zu beobachten ist, hervor. Frauen tragen weiterhin die Hauptlast der privaten Reproduktion, die sie nun zugleich in den Dienst der Öffentlichkeit zu stellen haben. Sie werden jetzt von staatlicher Seite in der Gestaltung ihres privaten Bereiches angesprochen: Waren sie vorher für die ihren verantwortlich, sind sie jetzt zusammen mit den Ihren für alle anderen mitverantwortlich. Der Staat greift so in den ihnen gesellschaftlich zugesprochenen Hoheitsbereich ein - in einer Art und Weise, die dem hervorgehenden Individualisierungsappell neoliberaler Schule in Teilen zuwiderläuft. So reihen sich die Corona-Maßnahmen zwar in Bezug auf eine höhere Belastung von Frauen in den neoliberalen Umbau ein, zeichnen sich jedoch durch eine für den neoliberalen Privatisierungsgedanken unbekannte Orientierungspflicht an das öffentliche Wohl aus.

\section{Der Widerspruch der Trennung von}

\section{privat und öffentlich}

Die These, dass im Zweifel Familie und Gesellschaft, Privates und Öffentliches, nicht nur unvereinbar, sondern gegeneinander gerichtet sind, wollen wir im Folgenden mit Rückgriff auf Hegel veranschaulichen, dessen Denken die Grundlagen und den historischen Beginn der bürgerlich-kapitalistischen Gesellschaft philosophisch nachzuverfolgen sucht. Wir stützen uns dafür auf seine Überlegungen zur Familie und ihren einzelnen Mitgliedern im Verhältnis zur Gemeinschaft ${ }^{20}$, die Hegel in seiner Antigone-Auslegung $^{21}$ in der Phänomenologie des Geistes (vgl. Hegel 1988: VI.A.a und VI.A.b) darbietet. Diese legt einen grundlegenden Widerspruch zwischen der Frau und der Gemeinschaft ${ }^{22}$ offen, der sich auch in der bürgerlich-kapitalistischen Gesellschaft findet und paradigmatisch in den Corona-Protesten der Frauen zum Ausdruck kommt. Wir kommen nun also zum gesellschaftsgefährdenden Aspekt, der in der Affirmation der vergeschlechtlichen Trennung von privater und öffentlicher Sphäre der bürgerlich-kapitalistischen Gesellschaft enthalten ist.

Zunächst lernen wir von Hegel Folgendes über die Rolle der Familie und ihrer Glieder (Frau, Mann, Sohn, Tochter) in der Gemeinschaft: Die Frau folgt den Gesetzen der Familie, sorgt sich um diese und ist ausschließlich ihren Angehörigen verpflichtet. Ihre Rolle besteht allein durch die

des Rechts (vgl. Hegel 1986). Gleichwohl finden wir besagte Auslegung zur Krisenbeschreibung von Frau und Gemeinschaft, von privater und öffentlicher Sphäre, geeigneter als die befriedete Welt der rechtlich verfassten Bürgerlichkeit der hegelschen Rechtsphilosophie, in der gesellschaftliche Widersprüche eingefangen sind und die Frau ganz in ihrer gesellschaftstragenden Rolle als

Familienverantwortliche aufgeht.

${ }^{22}$ Dieser Widerspruch zur Gemeinschaft findet sich weder beim Mann noch beim Sohn. 
Familie. Der Mann hingegen ist auf das Gemeinwesen, auf das Allgemeine, ausgerichtet. Um ein Mann zu werden, verlässt der Sohn die Familie bei Volljährigkeit und stellt sich in den Dienst der Öffentlichkeit, wohingegen die Tochter bei Volljährigkeit durch Heirat lediglich von der einen in die andere Familie wechselt, nun einer eigenen vorsteht und dadurch zur Frau wird. Der Widerspruch, auf den wir hinauswollen, wird deutlich, wenn Hegel daran erinnert, dass der Frau nicht nur die Aufgabe der Sorge um ihre Angehörigen zukommt, sondern sie es auch ist, die ihre Kinder auf ihre Rollen im Gemeinwesen vorbereitet. Dies bedeutet für sie, die ihren Sohn unter den Gesetzen der Familie erzogen hat, bei Volljährigkeit ebendiesen Sohn - und damit ihre Hauptaufgabe und den Grund ihrer gesellschaftlichen Existenz - an das Gemeinwesen zu verlieren. Durch Beeinflussung ihres Sohnes versucht die Frau zwar ihrem Verlust entgegenzuwirken und ihren Sohn auch in seiner öffentlichen Rolle zugunsten ihrer Familie zu beeinflussen und damit ihre Hoheit über den Sohn zu erhalten. Das Gemeinwesen setzt dieser Bewegung jedoch, nach Hegel, durch die Einberufung des Jünglings in den Krieg ein Ende, denn im Krieg verliert der Jüngling alles Private und ist nur noch Allgemeines. Der Krieg bricht die letzte Einflussmöglichkeit der Mutter auf den Sohn und damit ihre einzige Möglichkeit, durch ihn in der Öffentlichkeit mit rein privaten Zwecken zu agieren und seine Politik auf das Wohl ihrer Familie auszurichten.

${ }^{23}$ Wir kürzen das Zitat um "des Bewusstseins" und lassen damit Hegels Anliegen in der Phänomenologie des Geistes, eine "Geschichte des Bewusstseins" (Bonsiepen 1988: XXIX) zu schreiben, in den Hintergrund treten. Der Grund für dieses Vorgehen liegt in unserem Anspruch, weniger eine eigene Hegel-Interpretation zu liefern, als vielmehr einen hegelschen Gedanken für eine aktuelle

Gesellschaftsanalyse fruchtbar machen.
Nach Hegel agiert die Frau in Bezug auf ihren Sohn als Gefahr für die Gemeinschaft; eine Gefahr, die sich nur durch Krieg bannen lässt, aber nicht so, dass der Krieg sich gegen die Frau richtet, sondern so, dass er ihr den Sohn entreißt. Für Hegel erzeugt damit das Gemeinwesen „an der Weiblichkeit überhaupt seinen innern Feind“ (Hegel 1986: 314). Denn „[i]ndem das Gemeinwesen sich nur durch die Störung der Familienglückseligkeit und die Auflösung [...] in das allgemeine sein Bestehen gibt, erzeugt es sich an dem, was es unterdrückt und was ihm zugleich wesentlich ist" (Hegel 1986: 314). ${ }^{23}$ Die Frau richtet sich gegen das Gemeinwesen, sie steht im Widerspruch zu $\mathrm{ihm}$, und ist gleichzeitig sein integraler Bestandteil. Dieser Widerspruch - „die ewige Ironie des Gemeinwesens“ (Hegel 1986: 314) - ist von der Gemeinschaft selbst hervorgebracht. Die Gemeinschaft gibt der Frau erst und ausschließlich die Rolle der Sorge um ihre Familie. Der Krieg indes bildet die Lösung, die Aufhebung dieses Widerspruches, indem er ihn einseitig zugunsten der Gemeinschaft auflöst: Der Sohn wird der Frau entrissen, sie verliert ihn. Er wird gänzlich allgemeines Gut. ${ }^{24}$

Aus diesem kurzen Rückgriff auf Hegels Gedanken wollen wir vor allem eine Erkenntnis mitnehmen: Diejenigen, deren gesellschaftliche Rolle darin besteht, nur für die Familie zu leben, können sich im Zweifel gegen eben die Gesellschaft stellen, die ihnen diese Rolle zuwies. In der Hinsicht stehen Frau und Gesellschaft im Widerspruch zu einander. So, wie sich Hegels Frau gegen den Verlust ihrer Hoheit über

\footnotetext{
${ }^{24}$ Das Recht in Hegels Rechtsphilosophie bietet einen anderen Lösungsweg dieses Widerspruches an, einen, der auch das Private "zu seinem Recht" kommen lässt und der sich daher weit mehr als Krieg zur Befriedung des Widerspruches und der Frau anbietet.
} 
den Sohn wehrt, verteidigen die an den Corona-Protesten beteiligten Frauen ihren Hoheitsbereich der Sorge um die Angehörigen. Erstere sucht ihren Sohn weiterhin im Sinne ihrer Familie zu beeinflussen, letztere ihre Familie vor staatlichen Eingriffen abzuschirmen. Indem beide ihre Familie als höchsten Zweck ihrer Handlungen setzen, agieren sie im Zweifel gegen ihre Gesellschaften. Nämlich dann, wenn sich der familiäre nicht mit dem gesellschaftlichen Zweck deckt - bei Hegel zeigt sich das im Krieg, in der Corona-Pandemie, sehr viel schwächer, in der Ablehnung von staatlich verordneten Maßnahmen, die den gesundheitlichen Schutz der Gesellschaft bewirken sollen.

So gefährden die Frauen der Corona-Proteste wie Hegels Frau ihre Gesellschaft: durch die Affirmation und Verteidigung ihrer gesellschaftlichen Rolle.

\section{Fazit}

Am Protest der Frauen gegen bestimmte Corona-Maßnahmen lässt sich veranschaulichen, wie eine Affirmation gesellschaftlicher Zustände sich gegen eben die Gesellschaft richten kann, die diese Zustände hervorbringt. Unter genauerer Betrachtung zweier Themen der Corona-Proteste (das Impfen und die Sorge um die Angehörigen) deuten wir die breite Beteiligung von Frauen an den Corona-Protesten als Verteidigung einer Vorstellung von Natur und ihrer gesellschaftlich zugeschriebenen Hoheit über die private Sphäre. Sie nehmen die ihnen auferlegte gesellschaftliche Rollen des Naturwesens und der primär im Privaten Verhafteten als natürlich an und tragen zur anhaltenden romantischen Verklärung ihrer Rolle bei. So affirmieren sie die für die bürgerlich-kapitalistische
Gesellschaft konstitutive vergeschlechtliche Trennung von privater und öffentlicher Sphäre. Diese Affirmation kann sich, auch das zeigt sich beispielhaft in ihrem Protest, gegen die Gesellschaft richten, nämlich dann, wenn sie einen Angriff auf ihren Hoheitsbereich sehen und dadurch ihre gesellschaftliche Rolle auf dem Spiel steht. Dann richtet sich die Frau gegen die Gesellschaft, paradigmatisch indem sie staatliche Maßnahmen zum Schutz und Erhalt dieser Gesellschaft ablehnt.

So lässt sich unser Deutungsvorschlag wie folgt zusammenfassen: Die breite Beteiligung von Frauen an den Corona-Protesten kann als Ausdruck gesellschaftlicher Verhältnisse interpretiert werden, in denen ihre Affirmation zugleich ihre Gefährdung bedeuten kann. In Bezug auf die vergeschlechtlichte Trennung von öffentlicher und privater Sphäre zeigt sich der mit Hegel thematisierte Widerspruch von Frau und Gesellschaft. Dabei scheint es für die Frauen, die sich an den Corona-Protesten beteiligen, ebenso wie für Hegel ausgemacht, dass eine Antwort auf den Widerspruch von Frau und Gesellschaft, der sich auf die Trennung von privater und öffentlichen Sphäre gründet, für die Frau im Verteidigen ihrer gesellschaftlichen Rolle liegen muss. Sie bestätigen die herrschenden gesellschaftlichen Verhältnisse - die Geschlechterordnung der Polis ebenso wie die vergeschlechtlichte Sphärentrennung der bürgerlich-kapitalistischen Gesellschaft. 


\section{Referenzen}

Adamczak, Bini (2017) Beziehungsweise Revolution - 1917, 1968 und kommende. Berlin: Suhrkamp.

Alemann, Annette von/Beaufaÿs, Sandra/Kortendiek, Beate (Hrsg.). (2017). Alte neue Ungleichheiten? Auflösungen und Neukonfigurationen von Erwerbs- und Familiensphäre. GENDER Zeitschrift für Geschlecht, Kultur und Gesellschaft, Sonderheft 4. Opladen: Budrich Verlag. https://doi.org/10.3224/84740545

Alischer, Béatrice (2018). Das Geschlechterverhältnis in der Care-Debatte. Modernisierung oder Persistenz? In Maik Krüger (Hrsg.). Fürsorge-Relationen. Theoretische und empirische Sichtweisen auf Care (S. 17-37). Soziologiemagazin, Sonderheft 3. München: sozologiemagazin e.V.

Aulenbacher, Brigitte/Meuser, Michael/Riegraf, Birgit (2012). Geschlecht, Ethnie, Klasse im Kapitalismus - Über die Verschränkung sozialer Verhältnisse und hegemonialen Deutungen im gesellschaftlichen Reproduktionsprozess. Berliner Journal für Soziologie 22, S. 5-27. https://doi.org/10.1007/s11609-012-0181-4

Badinter, Elisabeth (1984). Die Mutterliebe. Geschichte eines Gefühls vom 17. Jahrhundert bis heute. München: dtv.

Becker-Schmidt, Regina/Knapp, Gudrun-Alexi (1987). Geschlechtertrennung - Geschlechterdifferenz. Suchbewegungen sozialen Lernens. Bonn: Verlag Neue Gesellschaft.

Berger, Stefan (2020). Erst kam die Flucht, dann Corona - oder: Welche Folgen werden die Corona-Bekämpfungsmaßnahmen für das Leben und Netzwerken von Geflüchteten in Deutschland haben? In Christian Stegbauer/Iris Clemens (Hrsg.). Corona-Netzwerke. Gesellschaft im Zeichen des Virus (S.187-195). Wiesbaden: Springer. https://doi.org/10.1007/978-3658-31394-4_18

Berlin, Isaiah (1999). The Roots of Romanticism. Princeton: Princeton University Press. https://doi.org/10.2307/j.ctt24hr5m

Bonsiepen, Wolfgang (1988). Einleitung. In Georg Wilhelm Friedrich Hegel. Phänomenologie des Geistes (S.IX-LXIII). Hamburg: Felix Meiner. https://doi.org/10.1017/s0263523200006212

Connell, Raewyn (2000). Der gemachte Mann. Konstruktion und Krise von Männlichkeiten. Opladen: Leske + Budrich.

Degele, Nina/Winker, Gabriele (2011). Intersektionalität als Beitrag zu einer gesellschaftstheoretisch informierten Ungleichheitsforschung. Berliner Journal für Soziologie 21, S.69-90.

Dreas, Susanne A. (2019). Zum Verhältnis von Gender und Care oder: Warum ist Sorgearbeit weiblich? In Ludger Kohlhoff (Hrsg.). Aktuelle Diskurse in der Sozialwirtschaft II. 
Perspektiven Sozialwirtschaft und Sozialmanagement (S. 223-239). Wiesbaden: Springer VS. https://doi.org/10.1007/978-3-658-25915-0_12

Durkheim, Emile (2014). Der Selbstmord. Frankfurt am Main: Suhrkamp.

Federici, Silvia (2015). Caliban und die Hexe. Frauen, der Körper und die ursprüngliche Akkumulation. Wien: Mandelbaum.

Fraser, Nancy (2016) Contradictions of Capital and Care. New Left Review 100, S. 99-117.

Frei, Nadine/Schäfer, Robert/Nachtwey, Oliver (2021): Die Proteste gegen die Corona-Maßnahmen. Eine soziologische Annäherung. FJSB, 2021 (34), S. 249-258. https://doi.org/10.1515/fjsb2021-0021

Hausen, Karin (2001). Die Polarisierung der »Geschlechtscharaktere«. Eine Spiegelung der Dissoziation von Erwerbs- und Familienleben. In Werner Conze (Hrsg.), Sozialgeschichte der Familie in der Neuzeit Europas (S. 363-393). Stuttgart: Klett-Cotta. https://doi.org/10.13109/9783666370250.19

Hegel, G. W. F. (1988). Phänomenologie des Geistes. Hamburg: Felix Meiner.

Hegel, G. W. F. (1986). Grundlinien der Philosophie des Rechts oder Naturrecht und Staatswissenschaft im Grundrisse. Frankfurt am Main: Suhrkamp. https://doi.org/10.1007/978-3-47605728-0_9608-1

Hobler, Dietmar/Lott, Yvonne/Pfahl, Svenja/Schulze Buschoff, Karin (2020). Stand der Gleichstellung von Frauen und Männern in Deutschland. WSI Report 56.

Honegger, Claudia (1989). »Weiblichkeit als Kulturform». Zur Codierung der Geschlechter in der Moderne. In Max Haller et.al. (Hrsg.). Kultur und Gesellschaft. Verhandlungen des 24. Deutschen Soziologentags, des 11. Österreichischen Soziologentags und des 8. Kongresses der Schweizerischen Gesellschaft für Soziologie in Zürich 1988 (S. 142-155). Frankfurt am Main/New York: Campus. https://doi.org/10.1007/978-3-642-83771-5_1

Honegger, Claudia (1992). Die Ordnung der Geschlechter. Die Wissenschaften vom Menschen und das Weib. Frankfurt am Main/New York: Campus. https://doi.org/10.7767/lhomme.1992.3.2.114

Honegger, Claudia/Heintz, Bettina (Hrsg.) (1984). Listen der Ohnmacht. Zur Sozialgeschichte weiblicher Widerstandsformen. Frankfurt am Main: Europäische Verlagsanstalt.

Klinger, Cornelia (1993). Romantik und Neue Soziale Bewegungen. Athenäum. Jahrbuch für Romantik 3, 223-244.

Knapp, Gudrun-Alexi/Klinger, Cornelia (2007). Achsen der Ungleichheit - Achsen der Differenz. Verhältnisbestimmungen von Klasse, Geschlecht, »Rasse«/Ethnizität. In Gudrun-Alexi Knapp/ Cornelia Klinger/ Birgit Sauer: Achsen der Ungleichheit. Zum Verhältnis von Klasse, Geschlecht und Ethnizität S. 19-41. Frankfurt/Main, New York: Campus. 
Kohlrausch, Bettina/Hoevermann, Andreas (2020). Soziale Ungleichheit und Einkommenseinbußen in der Corona-Krise - Befunde einer Erwerbstätigenbefragung. WSI Mitteilungen, 2020 (6), 485-492. https://doi.org/10.5771/0342-300x-2020-6-485

Kohlrausch, Bettina/Zucco, Aline (2020). Die Corona-Krise trifft Frauen doppelt - Weniger Erwerbseinkommen und mehr Sorgearbeit. WSI Policy Brief, 2020 (5).

Koos, Sebastian (2021). Die "Querdenker“. Wer nimmt an den Corona-Protesten teil und warum? Ergebnisse einer Befragung während der „Corona-Proteste“ am 4.10.2020 in Konstanz. http://nbn-resolving.de/urn:nbn:de:bsz:352-2-bnrddxo8opad0

Krüger, Jens Oliver/Krüger, Kathrin (2015). Skepsis im Entscheiden. Wie begründen impfskeptische Eltern ihre Impfentscheidungen? ZQF, $2015 \quad$ (1), 99-114. https://doi.org/10.3224/zqf.v16i1.22856

Mannheim, Karl (1984). Konservatismus. Ein Beitrag zur Soziologie des Wissens. Frankfurt am Main: Suhrkamp.

Marx, Karl [MEW 23] (1968). Das Kapital. Erster Band. Berlin: Dietz.

Meyer, Christiane (2002). Über die Bedeutung von Schutzimpfungen. Epidemiologie, Durchimpfungsraten, Programme. Bundesgesundheitsblatt - Gesundheitsforschung - Gesundheitsschutz, 2002 (45), 323-331. https://doi.org/10.1007/s00103-002-0398-z

Mies, Maria (2015) Patriarchat und Kapital. München: bge-verlag.

Möhring, Katja et.al. (2020). Die Mannheimer Corona-Studie: Schwerpunktbericht zu Erwerbstätigkeit und Kinderbetreuung. Abgerufen am 02. Februar 2021, von https://www.unimannheim.de/media/Einrichtungen/gip/Corona_Studie/2020-04-05_Schwerpunktbericht_Erwerbstaetigkeit_und_Kinderbetreuung.pdf

Nachtwey, Oliver/Schäfer, Robert/Frei, Nadine (2020). Politische Soziologie der Corona-Proteste. Universität Basel. https://doi.org/10.31235/osf.io/zyp3f

Neumann, Matthias/Winker, Gabriele (2019). Sorge und Solidarität. Von verbindender CarePolitik zur solidarischen Gesellschaft. Online-Publikation. https://care-revolution.org/wp-content/uploads/2019/09/09-Sorge_und_Solidarität.pdf (Stand 21.04.2021).

Petzold, Moritz Bruno/Plag, Jens/Ströhle, Andreas (2020). Umgang mit psychischer Belastung bei Gesundheitsfachkräften im Rahmen der Covid-19-Pandemie. Nervenarzt, 2020 (91), 417421. https://doi.org/10.1007/s00103-002-0398-z

Reich, Jennifer A. (2016). Calling the Shots. Why Partents Reject Vaccines. New York: University Press.

Rendtorff, Barbara/Riegraf, Birgit/Mahs, Claudia (Hrsg.). (2019). Struktur und Dynamik Un/Gleichzeitigkeiten im Geschlechterverhältnis. Wiesbaden: Springer VS. https://doi.org/10.1007/978-3-658-22311-3 
Riegraf, Birgit (2019). Care, Care-Arbeit und Geschlecht: gesellschaftliche Veränderungen und theoretische Auseinandersetzungen. In Beate Kortendiek/Birgit Riegraf/Katja Sabisch (Hrsg). Handbuch Interdisziplinäre Geschlechterforschung. Geschlecht und Gesellschaft (S. 762772). Wiesbaden: Springer VS. https://doi.org/10.1007/978-3-658-12496-0_172

Scheele, Alexandra/Wöhl, Stefanie (Hrsg.) (2018) Feminismus und Marxismus. Weinheim Basel: Beltz Juventa.

Simmel, Georg [GSG 6] (1989). Philosophie des Geldes. Frankfurt am Main: Suhrkamp.

Simmel, Georg (1985). Bruchstücke aus einer Psychologie der Frauen. In Heinz-Jürgen Dahme/Klaus Christian Köhnke (Hrsg.). Georg Simmel. Schriften zur Philosophie und Soziologie der Geschlechter (S. 177-182). Frankfurt am Main: Suhrkamp. https://doi.org/10.2307/2070505

Soiland, Tove (2018). Soziale Reproduktion und Neue Landnahme: ein feministischer Zugang. In Widersprüche 150, 85-110.

Soiland, Tove (2019). Ökonomisierung - Privatisierung: die verdeckte Unterseite neoliberaler Restrukturierungen und ihre Implikationen für die Geschlechterforschung. In Beate Kortendiek/Birgit Riegraf/Katja Sabisch (Hrsg). Handbuch Interdisziplinäre Geschlechterforschung. Geschlecht und Gesellschaft (S. 95-104). Wiesbaden: Springer. https://doi.org/10.1007/978-3-658-12496-0_13

Steinert, Janina/Ebert, Cara (2020). Gewalt an Frauen und Kindern in Deutschland während COVID-19-bedingten Ausgangsbeschränkungen. Abgerufen am 02. Februar 2021, von https://drive.google.com/file/d/19Wqpby9nwMNjdgO4_FCqqlfYyLJmBn7y/view

Villa, Paula-Irene (2020). Corona-Krise meets Care-Krise - Ist das systemrelevant? Leviathan, 2020 (3), 433-450. https://doi.org/10.5771/0340-0425-2020-3-433

Walgenbach, Katharina (2015). Geschlecht in gesellschaftlichen Transformationsprozessen. In Katharina Walgenbach/Anna Stach (Hrsg.). Geschlecht in gesellschaftlichen Transformationsprozessen (S. 21-51). Opladen: Budrich Verlag. https://doi.org/10.2307/j.ctvddzvpc.4

Weber, Marianne (2008). Die Frau und die objektive Kultur. In Ute Gerhard/Petra Pommerenke/Ulla Wischermann (Hrsg.). Klassikerinnen feministischer Theorie. Band 1 (S. 368383). Sulzbach: Ulrike Helmer Verlag.

Winker, Gabriele (2007). Traditionelle Geschlechterordnung unter neoliberalem Druck. Veränderte Verwertungs- und Reproduktionsbedingungen der Arbeitskraft. In Melanie Groß/Gabriele Winker (Hrsg.). Queer-Feministische Kritiken neoliberaler Verhältnisse (S. 1549). Münster: Unrast.

Zetkin, Clara (2008). Nur mit der proletarischen Frau wird der Sozialismus siegen. In Ute Gerhard/Petra Pommerenke/Ulla Wischermann (Hrsg.). Klassikerinnen feministischer Theorie. Band 1 (S. 189-200). Sulzbach: Ulrike Helmer Verlag. 


\section{Educating}

\section{Talents}

since 1460.

Universität Basel

Seminar für Soziologie

Petersgraben 27

4052 Basel

Switzerland

www.unibas.ch 